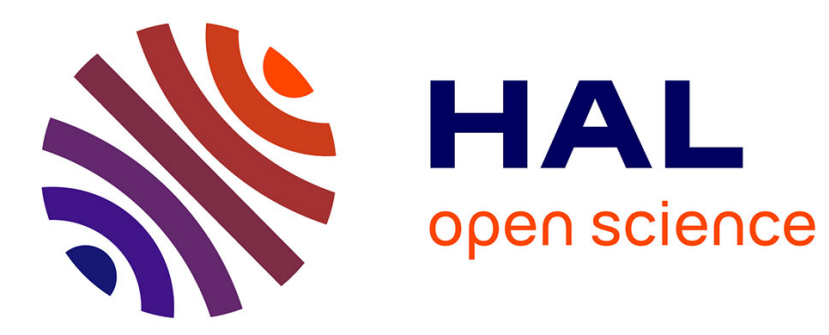

\title{
Robust motion planning for a heat rod process
}

\author{
Stephane Victor, Pierre Melchior, Rachid Malti, Alain Oustaloup
}

\section{To cite this version:}

Stephane Victor, Pierre Melchior, Rachid Malti, Alain Oustaloup. Robust motion planning for a heat rod process. Nonlinear Dynamics, 2016, 86 (2), pp.1271 - 1283. 10.1007/s11071-016-2963-2 . hal-01485003

\section{HAL Id: hal-01485003 https://hal.science/hal-01485003}

Submitted on 18 Jul 2017

HAL is a multi-disciplinary open access archive for the deposit and dissemination of scientific research documents, whether they are published or not. The documents may come from teaching and research institutions in France or abroad, or from public or private research centers.
L'archive ouverte pluridisciplinaire HAL, est destinée au dépôt et à la diffusion de documents scientifiques de niveau recherche, publiés ou non, émanant des établissements d'enseignement et de recherche français ou étrangers, des laboratoires publics ou privés. 


\title{
Robust motion planning for a heat rod process
}

\author{
Stéphane Victor • Pierre Melchior • Rachid Malti · Alain Oustaloup
}

Received: date / Accepted: date

\begin{abstract}
First, heat rod models linking temperature to heat flux density are obtained from system identification using fractional order systems. Then, motion planning of the nominal system is obtained through an open-loop control stemming from flatness principles. Usually, each model should have its own control reference in order to follow a desired output reference. Thanks to a third generation CRONE controller, the nominal control reference is sufficient, and robust control is also guaranteed regarding model uncertainties and input/output disturbances. Robust motion planning is held on a real heat experiment and comparison between CRONE and PID controllers are proposed on this test bench.
\end{abstract}

Keywords thermal system - fractional order system · system identification · differential flatness · motion planning $\cdot$ robust control

\section{Introduction}

Fractional (or non-integer) operator is a modeling tool that enables explaining diffusive phenomena thanks to its long memory character and parametric compacity: thermal diffusive phenomena [29][23], charge diffusion in acid batteries [34][35], in rheology [17], Foucault currents inside rotor bars in induction machines [7]...

Fractional order system (FOS) identification was initiated in the nineties [10][4][15]. Refined Instrumental Variables for Continuous system (rivc) [38], and

Université de Bordeaux - Bordeaux INP/Enseirb-Matmeca IMS, UMR 5218 CNRS

351 cours de la Libération, F 33405 Talence cedex, France tel : + (33) 540003627 - fax : + (33)540006644

E-mail: surname.name@ims-bordeaux.fr its simplified version (srivc) when the additive measurement noise is white [16] have been extended for FOS [36]. In the usual case of rational model system identification, where only coefficients are estimated, the model order remains unchanged, whereas in the fractional model case, estimating the differentiation orders in an iterative algorithm changes the model order at each iteration. Also, the oosrivcf algorithm is recalled for all parameter estimation [36]. The latest version of the CRONE toolbox proposes a tutorial on fractional system identification (http://cronetoolbox.imsbordeaux.fr).

In motion planning, a suitable approach consists in using the flatness-based trajectory design (see [14][20] and the references therein) adapted to FOSs [37]. Recall that a system described by ordinary differential equations is said to be differentially flat if and only if there exists an output vector (flat output) of the same dimension as the control vector, such that all system variables can be expressed as functions of this output and its derivatives. This motion planning is generally carried out in open-loop and robust control may be achieved with a suitable control feedback. Several industrial applications have emerged in control theory, such as $H_{\infty}$ state feedback control of a bridge seismic response [11], $H_{\infty}$ consensus method for a network of nonlinear multiagent system [1], trajectory tracking control of cooperative multiple mobile cranes [33], pollutant reduction of a turbocharged diesel engine [19] and so on. Here, a CRONE controller [30] is used to guarantee stability degree, path tracking and disturbance rejection.

The contributions are on the heat experiment:

- solving the heat equation leads to a fractional model [31], therefore an optimal estimator is proposed for FOS identification (see $§ 2.3$ );

- open-loop motion control is proposed using flatness; 
- closed-loop robust path tracking is held through the design of a unique CRONE controller.

After recalling fractional calculus in section 1, section 2 presents the heat system modeling. Robust motion control is developed in section 3 . In section 4 , the experimental results are given, to finally conclude in section 5 .

\subsection{Fractional derivative}

Let $\gamma \in \mathbb{R}_{+}, n=\lceil\gamma\rceil=E(\gamma)+1=\min \{k \in \mathbb{N} \mid k>\gamma\}$, where $\lceil$.$\rceil (resp. E) is the ceiling operator (resp. the$ integer part), and $\nu \in[0,1$ [ given by $\nu=n-\gamma$. Let a be a given arbitrary real number and $f \in \mathbb{C}^{\infty}([\mathrm{a},+\infty[)$, the set of infinitely continuously differentiable functions from $[\mathrm{a},+\infty[$ to $\mathbb{R}$.

The Riemann-Liouville derivative of function $f$ at time $t$ of order $\gamma=n-\nu$ is defined as [28]:

$$
\begin{aligned}
\mathbf{D}_{\mathrm{a}}^{\gamma} f(t) & =\mathbf{D}^{n}\left(\mathbf{I}_{\mathrm{a}}^{\nu} f(t)\right) \\
& \triangleq\left(\frac{d}{d t}\right)^{n}\left(\frac{1}{\Gamma(\nu)} \int_{\mathrm{a}}^{t} \frac{f(\tau) d \tau}{(t-\tau)^{1-\nu}}\right)
\end{aligned}
$$

where $\mathbf{D}=\frac{d}{d t}$ is the differentiation operator, $\mathbf{I}_{\mathrm{a}}^{\nu} f(t)$ is called the fractional primitive of $f$ and the Euler's $\Gamma$ function, defined by:

$\Gamma(x)=\int_{0}^{\infty} e^{-t} t^{x-1} d t, \quad \forall x \in \mathbb{R}^{*} \backslash \mathbb{N}^{-}$,

is the generalized factorial $(\forall n \in \mathbb{N}, \Gamma(n+1)=n !)$. Let be

$\mathfrak{H}_{\mathrm{a}} \triangleq\left\{f: \mathbb{R} \mapsto \mathbb{R} \mid f \in \mathbb{C}^{\infty}([\mathrm{a},+\infty[), f(t)=0, \forall t \leq \mathrm{a}\}\right.$.

As a consequence of Propositions 15 of [37] (or e.g. [31, chap, 2, p. 57]), $\mathbf{D}_{a}^{\gamma}$ is an endomorphism from $\mathfrak{H}_{a}$ to itself and $\mathfrak{H}_{\text {a }}$ may be considered as the domain of $\mathbf{D}_{\mathrm{a}}^{\gamma}$. For simplicity sake, a will be equal to 0 , therefore, the notation $\mathbf{D}^{\gamma}$ is used in place of $\left.\mathbf{D}_{\mathrm{a}}^{\gamma}\right|_{\mathfrak{H}_{\mathbf{a}}}$.

\section{$1.2 \mathbf{D}^{\gamma}$-polynomial matrices}

$\mathbf{D}^{\gamma}$-polynomials are polynomials of the indeterminate $\mathbf{D}^{\gamma}$ with real coefficients, of the form $\sum_{k=0}^{K} c_{k} \mathbf{D}^{k \gamma} \cdot \mathbb{R}\left[\mathbf{D}^{\gamma}\right]$ denotes the set of $\mathbf{D}^{\gamma}$-polynomials; the reader may immediately verify that $\left(\mathbb{R}\left[\mathbf{D}^{\gamma}\right],+, \times\right)$ is a (commutative) principal ideal domain ${ }^{1}$.

Interpreting the fractional derivative operator as a Mikusiński's operator as in [6], a system theoretic approach has been developed by [13] where the field of

\footnotetext{
1 The main properties of $\mathbb{R}\left[\mathbf{D}^{\gamma}\right]$ are recalled in [37].
}

Mikusiński's operators $\mathcal{M}$ is defined as the field of fractions of the commutative integral domain $\mathcal{C}$ of continuous functions defined over $[0, \infty[$ endowed with the addition and convolution product $[27,13] . \mathcal{M}$ can also be considered as an $\mathbb{R}\left[s^{\gamma}\right]$-module, where $s^{\gamma}$ is the Laplace operator associated to $\mathbf{D}^{\gamma}$.

If $p, q \in \mathbb{N}$, call $\mathbb{R}\left[\mathbf{D}^{\gamma}\right]^{p \times q}$ the set of $\mathbf{D}^{\gamma}$-polynomial matrices of size $(p \times q)$. When $p=q$, the group of unimodular $\mathbf{D}^{\gamma}$-polynomial matrices, $G L_{p}\left(\mathbb{R}\left[\mathbf{D}^{\gamma}\right]\right)$, defines the set of invertible (square) $\mathbf{D}^{\gamma}$-polynomial matrices whose inverse is also a $\mathbf{D}^{\gamma}$-polynomial matrix. $I_{p}$ denotes the $p \times p$ identity matrix and by $0_{p \times q}$ the $p \times q$ zero matrix.

Theorem 1 (Smith diagonal decomposition [9]) Given $A \in \mathbb{R}\left[\mathbf{D}^{\gamma}\right]^{p \times q}$, with $p \leq q$ (resp. $p \geq q$ ), there exist two matrices $S \in G L_{p}\left(\mathbb{R}\left[\mathbf{D}^{\gamma}\right]\right)$ and $T \in$ $G L_{q}\left(\mathbb{R}\left[\mathbf{D}^{\gamma}\right]\right)$ such that:

$S A T=\left[\begin{array}{ll}\Delta & 0_{p, q-p}\end{array}\right] \quad\left(\right.$ resp. $\left.=\left[\begin{array}{c}\Delta \\ 0_{p-q, q}\end{array}\right]\right)$,

where $\Delta=\operatorname{diag}\left\{\delta_{1}, \ldots, \delta_{\sigma}, 0, \ldots, 0\right\} \in \mathbb{R}\left[\mathbf{D}^{\gamma}\right]^{p \times p}$ (resp. $\left.\mathbb{R}\left[\mathbf{D}^{\gamma}\right]^{q \times q}\right)$. The integer $\sigma \leq \min (p, q)$ is the rank of $A$ and every non zero $\mathbf{D}^{\gamma}$-polynomial $\delta_{i}$, for $i=1, \ldots, \sigma$, is a divisor of $\delta_{j}$ for all $\sigma \geq j \geq i$.

Definition 1 Hyper-regularity [37]. Given a matrix $A \in \mathbb{R}\left[\mathbf{D}^{\gamma}\right]^{p \times q}$, it is said that $A$ is hyper-regular if, and only if, in (4), one has $\Delta=I_{\min (p, q)}$.

A straightforward adaptation of [3, Section II.C] to $\mathbf{D}^{\gamma}$-polynomial matrices reads:

Proposition 1 A matrix $A \in \mathbb{R}\left[\mathbf{D}^{\gamma}\right]^{p \times q}$, (i) with $p<q$ is hyper-regular if, and only if, there exists right-inverse $T \in G L_{q}\left(\mathbb{R}\left[\mathbf{D}^{\gamma}\right]\right)$ such that

$A T=\left[\begin{array}{ll}I_{p} & 0_{p \times(q-p)}\end{array}\right] ;$

(ii) with $p \geq q$ is hyper-regular if, and only if, there exists a left-inverse $S \in G L_{p}\left(\mathbb{R}\left[\mathbf{D}^{\gamma}\right]\right)$ such that

$S A=\left[\begin{array}{c}I_{q} \\ 0_{(p-q) \times q}\end{array}\right]$.

\section{Heat rod model}

\subsection{Plant description}

A cylindrical aluminium rod of radius $R_{b a r}=1 \mathrm{~cm}$ and of length $40 \mathrm{~cm}$ is considered where a heating resistor is glued at one end. To ensure unidirectional heat transfer, the entire surface of the rod is insulated by a foam. The input signal is a heat flux generated by the resistor and the output signal is the temperature measured 


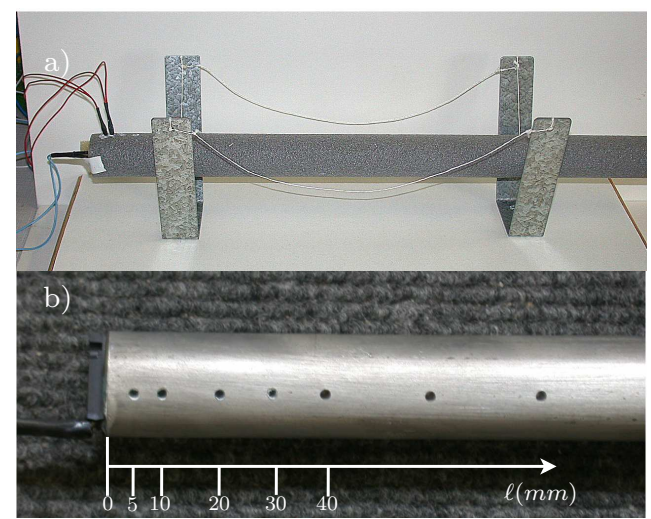

Fig. 1 a) Insulated aluminium rod heated by a resistor b) Measurement slots for sensor positions

at a distance $\ell$ from the heated end using a platinum probe (see Figure 1). Also, the process is constituted of a heating device and therefore, there is no cooling process to lower the temperature of the rod. However, due to heat leakage, the temperature of the thermal process naturally decreases when no heat density flux is injected.

From the heat equation, $[31,5]$ proved that the exact solution of the heat transfer linking the temperature to the heat flux density is expressed as a fractional model (see $§ 2.2$ and $§ 2.4$ ). Moreover, [24] showed that a rational model can be less accurate than a fractional model unless having higher number of parameters (as it happens twice the number of parameters).

Data are collected with a sampling period $T_{s}$ set to $0.5 \mathrm{~s}$. The number of samples is assumed large enough to guarantee convergence of the estimated parameters to the true ones. By injecting a constant flux density of $5.2 \mathrm{~kW} . \mathrm{m}^{-2}$, the system is driven to a steady-state (e.g. temperature of $42.2^{\circ} \mathrm{C}$ at $\ell=10 \mathrm{~mm}$ ). Then, to guarantee convergence of the identification procedure, a sufficiently persistent pseudo random binary sequence is applied with a variation of $\pm 5.2 \mathrm{~kW} . \mathrm{m}^{-2}$ around that constant flux density of $5.2 \mathrm{~kW} \cdot \mathrm{m}^{-2}$. The input density flux and the output temperature data are pretreated to eliminate the steady-state and delay (see Figure 2).

\subsection{Problem formulation}

A fractional differential equation can be written as:

$$
\begin{aligned}
& y(t)+a_{1} \mathbf{D}^{\alpha_{1}} y(t)+\cdots+a_{N} \mathbf{D}^{\alpha_{N}} y(t)= \\
& b_{0} \mathbf{D}^{\beta_{0}} u(t)+b_{1} \mathbf{D}^{\beta_{1}} u(t)+\cdots+b_{M} \mathbf{D}^{\beta_{M}} u(t),
\end{aligned}
$$

where $u(t) \in \mathfrak{H}_{0}$ is the input, $y(t) \in \mathfrak{H}_{0}$ is the noise-free output, $\left(a_{j}, b_{i}\right) \in \mathbb{R}^{2}$, and the differentiation orders $0<$
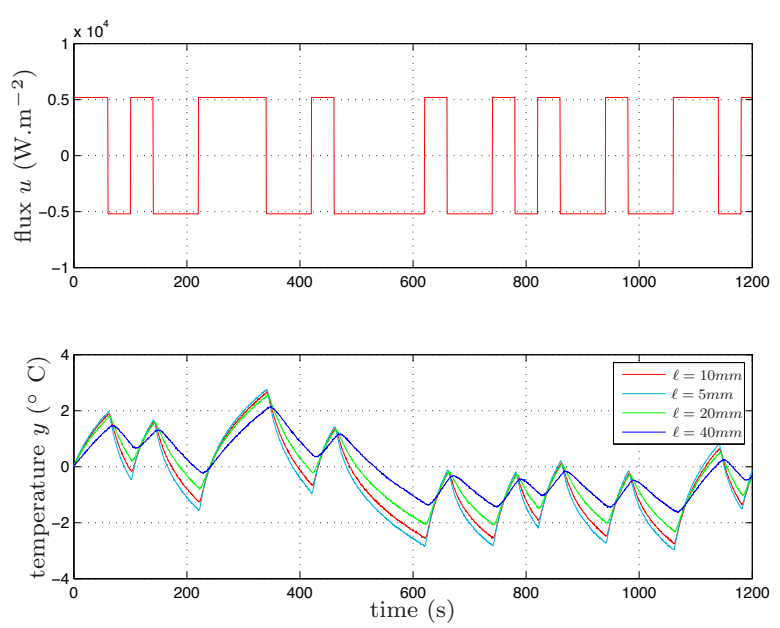

Fig. 2 Input/output signals (after steady state removal (temperature $\left(e . g .42 .2^{\circ} \mathrm{C}\right.$ at $\left.\ell=10 \mathrm{~mm}\right)$ and heat flux density of $\left.5.20 \mathrm{~kW} \cdot \mathrm{m}^{-2}\right)$ )

$\alpha_{1}<\ldots<\alpha_{N}, 0 \leq \beta_{0}<\ldots<\beta_{M}$, are ordered noninteger positive numbers for identifiability purposes.

It is assumed that the input $u(t)$ and the noise-free output $y(t)$ are related by differential equation (5), or also in a compact model form:

$G(\mathbf{D})=\frac{B(\mathbf{D})}{A(\mathbf{D})}=\frac{\sum_{i=0}^{M} b_{i} \mathbf{D}^{\beta_{i}}}{1+\sum_{j=1}^{N} a_{j} \mathbf{D}^{\alpha_{j}}}$,

$B(\mathbf{D})$ and $A(\mathbf{D})$ are assumed coprime, and the system asymptotically stable.

The quasi-stationary input signal $\{u(t), 0 \leq t \leq$ $\left.T_{\text {final }}\right\}$ applied to the system is persistently exciting, and gives rise to an output signal $\left\{y(t), 0 \leq t \leq T_{\text {final }}\right\}$. The noise-free output $y$ is supposed to be corrupted by an additive white measurement noise $\xi$, normally distributed with zero mean and $\sigma^{2}$ variance. The complete equation can be written as:

$\left\{\begin{array}{c}y(t)=G(\mathbf{D}) u(t) \\ y^{*}\left(t_{k}\right)=y\left(t_{k}\right)+\xi\left(t_{k}\right)\end{array}\right.$

where $y^{*}\left(t_{k}\right)$ is the sampled value of the unobserved noisy output $y(t)$. Given the discrete-time sampled nature of the data, the usual assumption is that a discretetime noise $\xi\left(t_{k}\right)$ is associated with the sampled data.

When the model (6) is used, the parameter vector

$\theta=\left[\begin{array}{l}\rho \\ \gamma\end{array}\right]$

is composed of a vector of $N+M+1$ coefficients,

$\rho=\left[b_{0}, b_{1}, \ldots, b_{M}, a_{1}, \ldots, a_{N}\right]^{\mathrm{T}}$, 
and a vector of $N+M+1$ differentiation orders,

$\gamma=\left[\beta_{0}, \ldots, \beta_{M}, \alpha_{1}, \ldots, \alpha_{N}\right]^{\mathrm{T}}$.

$N+M+1$ differentiation orders and $N+M+1$ coefficients need to be estimated. Moreover, if $N$ and $M$ are high, the number of local minima may increase and the nonlinear optimization algorithms may fail to converge to the global minimum. To reduce the number of parameters, a commensurate model can be chosen:

$G(\mathbf{D})=\frac{\sum_{i=0}^{M} b_{i} \mathbf{D}^{i \nu}}{1+\sum_{j=1}^{N} a_{j} \mathbf{D}^{j \nu}}$,

where the parameter vector $\theta$ in (8) is then reduced to the $N+M+1$ coefficients as in (9) added to a single commensurate differentiation order:

$\gamma=\nu$

In this case, the searching domain of the commensurate order is held for $\nu \in(0,2)$, corresponding to the stability domain (see e.g. Matignon's theorem [26]).

\subsection{System identification}

The order-optimization-srivcf algorithm (oosrivcf) uses the srivcf algorithm [25,36] for coefficient estimation along with a gradient-based algorithm for differentiation order estimation. Either a commensurate order for model (11) or all differentiation orders for (6), are estimated. The $\ell_{2}$-norm estimation problem is:

$J=\frac{1}{2}\|\varepsilon(t, \theta)\|^{2}$,

where the output error $\varepsilon(t, \theta)=y^{*}(t)-G(\mathbf{D}, \theta) u(t)$, with respect to the vector $\gamma$, as in (10) or (12).

A Gauss-Newton algorithm [22] is used for differentiation order estimation.

\section{Algorithm 1 oosrivef algorithm}

Inputs: The model $G\left(D, \theta^{0}\right)$ as in (6) or (11) and the input and output signals $\left(u(t), y^{*}(t)\right)$

Outputs: Model $\hat{G}(\mathbf{D}, \hat{\theta})$ with covariance matrix $\hat{\mathbf{P}}_{\theta}$ Procedure:

\section{Initialization}

iter $=0$

Initialize $\gamma^{0}$ and compute $\rho^{0}$ with the srivcf. From $\theta^{0}=\left[\rho^{0}, \gamma^{0}\right]$, compute $J\left(\theta^{0}\right)$ as in (13).
2. Gauss-Newton optimization

for iter $=1$ to convergence

Initialize the factor $\lambda=\Lambda$ (usually $=1)$

do

(i) Refine the order estimate:

$\gamma^{\text {iter }}=\gamma^{\text {iter-1 }}-\left.\lambda\left[H^{-1} \frac{\partial J}{\partial \gamma}\right]\right|_{\gamma=\gamma^{\text {iter }-1}}$

where the gradient is defined as

$\frac{\partial J}{\partial \gamma}=\left.\varepsilon^{\mathrm{T}}\left[\frac{\partial \varepsilon}{\partial \gamma}\right]\right|_{\gamma=\gamma^{\mathrm{iter}-1}}$

and the approximated Hessian $H$ as

$$
H=\left.\left.\left[\frac{\partial \varepsilon}{\partial \gamma}\right]^{\mathrm{T}}\right|_{\gamma=\gamma^{\text {iter }-1}}\left[\frac{\partial \varepsilon}{\partial \gamma}\right]\right|_{\gamma=\gamma^{\text {iter }-1}}
$$

(ii) Compute $\rho^{\text {iter }}$ using the srivcf.

(iii) Evaluate $J\left(\theta^{\text {iter+1 }}\right)$ as in (13).

(iv) $\lambda=\lambda / 2$

while $J\left(\theta^{\text {iter }+1}\right)>J\left(\theta^{\text {iter }}\right)$

iter $=$ iter +1

end for

3. Parametric error estimation

Estimate the covariance matrix $\hat{\mathbf{P}}_{\theta}$ from (14).

By assuming that the oosrivef algorithm converges in the sense that $\hat{\theta} \rightarrow \theta$, then an estimate of the covariance matrix can be obtained on the basis of the Gauss-Newton algorithm [22, Theorem 9.1] by:

$\hat{\mathbf{P}}_{\theta}=\hat{\sigma}^{2} \mathcal{H}^{-1}$

where $\hat{\sigma}^{2}$ is, as previously, the empirical estimate of noise variance and $\mathcal{H}$ is the approximate Hessian computed towards all the estimated parameters:

$\mathcal{H}=\frac{\partial \varepsilon^{\mathrm{T}}}{\partial \theta} \frac{\partial \varepsilon}{\partial \theta}=\frac{\partial \varepsilon^{\mathrm{T}}}{\partial\left[\rho^{\mathrm{T}} \gamma^{\mathrm{T}}\right]^{\mathrm{T}}} \frac{\partial \varepsilon}{\partial\left[\rho^{\mathrm{T}} \gamma^{\mathrm{T}}\right]^{\mathrm{T}}}$,

with $\frac{\partial \varepsilon}{\partial \gamma}$ defined as

$\frac{\partial \varepsilon}{\partial \gamma}=\left[-\frac{\partial \hat{y}}{\partial \beta_{0}}, \ldots,-\frac{\partial \hat{y}}{\partial \beta_{M}},-\frac{\partial \hat{y}}{\partial \alpha_{1}}, \ldots,-\frac{\partial \hat{y}}{\partial \alpha_{N}}\right]^{\mathrm{T}}$,

when all differentiation orders are estimated, or as:

$\frac{\partial \varepsilon}{\partial \gamma}=\frac{\partial \varepsilon}{\partial \nu}=-\frac{\partial \hat{y}}{\partial \gamma}$

when the commensurate order is estimated, and

$\frac{\partial \varepsilon}{\partial \rho}=-\left[\frac{\partial \hat{y}}{\partial b_{0}}, \ldots, \frac{\partial \hat{y}}{\partial b_{M}}, \frac{\partial \hat{y}}{\partial a_{1}}, \ldots, \frac{\partial \hat{y}}{\partial a_{N}}\right]^{\mathrm{T}}$, 


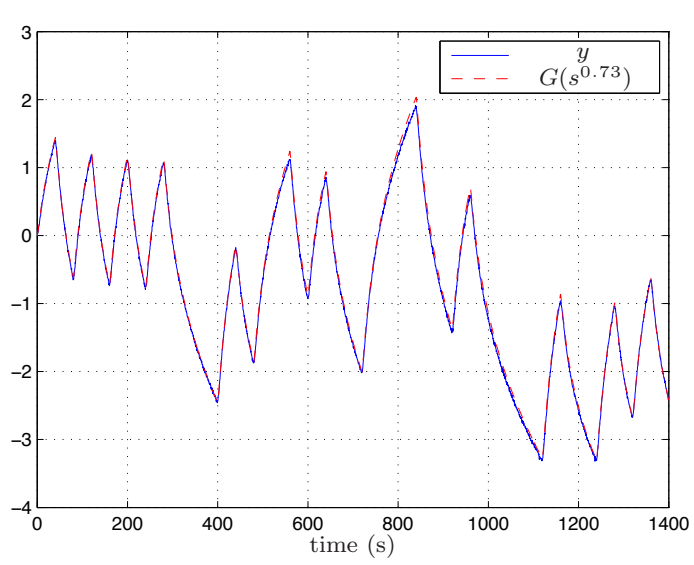

Fig. 3 Time responses of the estimated and true temperature outputs on validation data

where

$$
\begin{aligned}
\frac{\partial \hat{y}}{\partial b_{i}} & =\frac{\hat{\mathbf{D}}^{\hat{\beta}_{i}}}{1+\sum_{j=1}^{N} \hat{a}_{j} \mathbf{D}^{\hat{\alpha}_{j}}} u(t), \forall i=0, \ldots, M, \\
\frac{\partial \hat{y}}{\partial a_{k}} & =-\frac{\sum_{i=0}^{M} \hat{b}_{i} \mathbf{D}^{\hat{\beta}_{i}+\hat{\alpha}_{k}}}{\left(1+\sum_{j=1}^{N} \hat{a}_{j} \mathbf{D}^{\hat{\alpha}_{j}}\right)^{2}} u(t), \forall k=1, \ldots, N .
\end{aligned}
$$

\subsection{Black box identification}

Since the aluminium rod reaches a steady-state temperature, the insulation is flawed: the hypothesis of a commensurate order of 0.5 or an integrator as in the physical model [24] are unfit. After trial and error, the selected model is as the following with $M=1$ and $N=3$ :

$G(s)=\frac{b_{1} s^{\nu}+b_{0}}{a_{3} s^{3 \nu}+a_{2} s^{2 \nu}+a_{1} s^{\nu}+1} \times e^{-\tau s}$,

where a time delay $\tau$ is noticed between the heat density flux and the temperature measurement.

With the pseudo random binary sequence as explained in section $\S 2.1$ and with different temperature sensor positions (see Figure 1), all parameter models have been estimated with the oosrivcf algorithm (see algorithm 1): the nominal model is the one at $\ell=$ $10 \mathrm{~mm}$, the other models being estimated at $\ell=5,20$ and $40 \mathrm{~mm}$. After an initial differentiation order set to $\nu=0.6$, the algorithm converges to $\nu=0.73$ for the nominal model. The validation data for the nominal model, plotted on Figure 3, enable us to validate the model.

\begin{tabular}{|c|c|c|c|}
\hline$\ell$ & $\operatorname{model} G\left(s^{\nu}, \ell\right)$ & $\nu$ & $\tau$ \\
\hline 5 & $\frac{7.2010^{-4} s^{\nu}+5.4010^{-3}}{431.51 s^{3 \nu}+159.41 s^{2 \nu}+113.70 s^{\nu}+1} e^{-s}$ & 0.53 & 1 \\
\hline 10 & $\frac{2.9210^{-1} s^{\nu}+6.1110^{-3}}{25940.2 s^{3 \nu}+13038.7 s^{2 \nu}+561.66 s^{\nu}+1} e^{-2 s}$ & 0.73 & 2 \\
\hline 20 & $\frac{-1.9610^{-2} s^{\nu}+2.0310^{-2}}{1102.74 s^{2 \nu}+367.21 s^{\nu}+1} e^{-3.5 s}$ & 0.50 & 3.5 \\
\hline 40 & $\frac{-2.2910^{-2} s^{\nu}+3.8810^{-2}}{6953.97 s^{2 \nu}+1172.5 s^{\nu}+1} e^{-10 s}$ & 0.57 & 10 \\
\hline
\end{tabular}

Table 1 Identified models with different sensor positions ( $\ell$ in $\mathrm{mm}$ and $\tau$ in $\mathrm{s}$ )

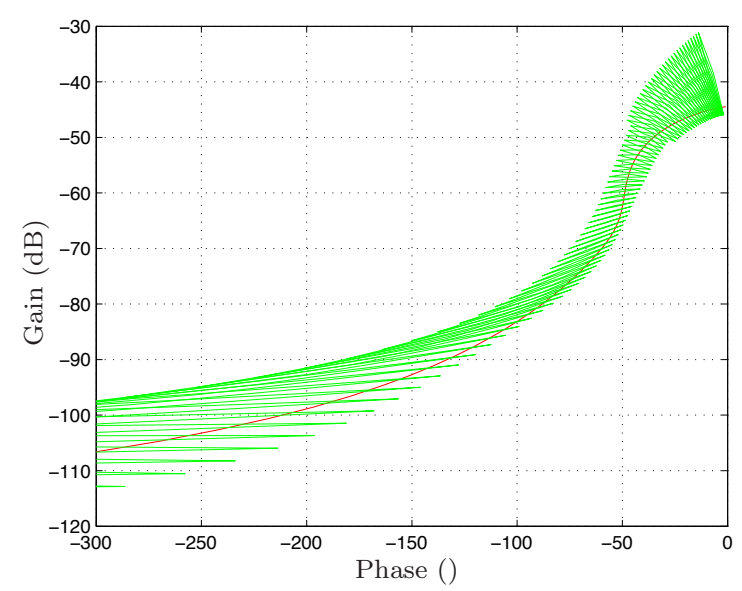

Fig. 4 Black-Nichols diagram of the nominal model (-) with uncertainties $(\downarrow)$

The models identified with the oosrivcf algorithm are presented in Table 1. This table shows that there are parametric variations for the coefficients, the commensurate order and the delay. Moreover, the longer the distance $\ell$, the higher the delay. The frequency responses of the identified models are plotted on Figure 4, which shows a high range of gain and phase uncertainties.

\section{Robust motion control}

\subsection{Linear fractionally flat systems}

System (11) can be put in the following representation

$A x=B u$

with state $x$ of dimension $n \times 1$, input $u$ of dimension $m \times 1, A \in \mathbb{R}\left[\mathbf{D}^{\gamma}\right]^{n \times n}$ and $B \in \mathbb{R}\left[\mathbf{D}^{\gamma}\right]^{n \times m}$. $B$ is assumed to be of rank $m$, with $1 \leq m \leq n$. Let us introduce $F \triangleq[A-B] \in \mathbb{R}\left[\mathbf{D}^{\gamma}\right]^{n \times(n+m)}$. For system (22), consider (see e.g. $[12,32]$ ): 
- its behavior ker $F$, where the kernel is taken w.r.t. the signal space $\mathfrak{H}_{0}$ defined in (3), i.e. the set

$$
\left\{\left[\begin{array}{l}
x \\
u
\end{array}\right] \in\left(\mathfrak{H}_{0}\right)^{n+m} \mid F\left[\begin{array}{l}
x \\
u
\end{array}\right]=0\right\} ;
$$

- and its system module $\mathfrak{M}_{F}$, i.e. the quotient module

$$
\mathfrak{M}_{F}=\mathbb{R}\left[\mathbf{D}^{\gamma}\right]^{1 \times(n+m)} / \mathbb{R}\left[\mathbf{D}^{\gamma}\right]^{1 \times n} F,
$$

where $\mathbb{R}\left[\mathbf{D}^{\gamma}\right]^{1 \times(n+m)}$ is the set of row vectors with components in $\mathbb{R}\left[\mathbf{D}^{\gamma}\right]$ and where $\mathbb{R}\left[\mathbf{D}^{\gamma}\right]^{1 \times n} F$ is the module generated by the rows of matrix $F$.

Assuming that $F$ has full (left) row rank, system (22) reads:

$F\left[\begin{array}{l}x \\ u\end{array}\right]=0$.

The definition of fractional flatness is detailed in [37] and is based on the notion of defining matrices in the spirit of [21] and [2].

Definition 2 The system (24) is called fractionally flat if, and only if, there exist matrices $P \in \mathbb{R}\left[\mathbf{D}^{\gamma}\right]^{m \times(n+m)}$ and $Q \in \mathbb{R}\left[\mathbf{D}^{\gamma}\right]^{(n+m) \times m}$ such that

$Q\left(\mathfrak{H}_{0}\right)^{m}=\operatorname{ker} F \quad$ and $\quad P Q=I_{m}$.

In other words, there exists a matrix $P$ with rightinverse $Q$ over the ring $\mathbb{R}\left[\mathbf{D}^{\gamma}\right]$ such that, for all $(x, u)$ satisfying $F\left[\begin{array}{l}x \\ u\end{array}\right]=0$, one has $y=P\left[\begin{array}{l}x \\ u\end{array}\right]$ and $\left[\begin{array}{l}x \\ u\end{array}\right]=$ $Q y$. The variable $y$, taking its values in $\left(\mathfrak{H}_{0}\right)^{m}$, is called fractionally flat output and the matrices $P$ and $Q$ are called defining matrices.

Theorem 2 One has the following equivalences [2, 37]:

(i) system (24) is fractionally flat;

(ii) the system module $\mathfrak{M}_{A, B}$ is free;

(iii) the matrix $F$ is hyper-regular over $\mathbb{R}\left[\mathbf{D}^{\gamma}\right]$.

In practice, it may be convenient to distinguish state and input, and for linear controllable time-invariant systems, a set of flat outputs may be obtained via Brunovský's canonical form (see e.g. $[8,18]$ ) and do not depend on the input $u$. This fractional 0 -flatness property reads: there exist $P_{1} \in \mathbb{R}\left[\mathbf{D}^{\gamma}\right]^{m \times n}$ and $Q_{1} \in \mathbb{R}\left[\mathbf{D}^{\gamma}\right]^{n \times m}$ such that $y=P_{1} x, x=Q_{1} y$, and $P_{1} Q_{1}=I_{m}$.

\section{Definition 3}

- A system is called fractionally $k$-flat, with $k \geq 1$, if and only if the maximal degree of the matrix $P\left[\begin{array}{c}0_{n, m} \\ I_{m}\end{array}\right]$ is equal to $k-1$. In this case, the output $y$ is called fractionally $k$-flat output.
- It is said fractionally 0-flat if $P\left[\begin{array}{c}0_{n, m} \\ I_{m}\end{array}\right]=0_{m}$, the output $y$ being called fractionally 0 -flat output.

Fractional 0-flatness is equivalent to the existence of $P$ and $Q$ such that $P=\left[\begin{array}{ll}P_{1} & 0_{m}\end{array}\right]$ with $P_{1} \in \mathbb{R}\left[\mathbf{D}^{\gamma}\right]^{m \times n}$ and $P_{1} Q_{1}=I_{m}$ where $Q_{1} \triangleq\left[I_{n} 0_{n, m}\right] Q$.

Lemma 1 (Elimination [37]) If $B$ is hyper-regular, there exists a unimodular matrix $M \in \mathbb{R}\left[\mathbf{D}^{\gamma}\right]^{n \times n}$ such that $M B=\left[\begin{array}{c}I_{m} \\ 0_{(n-m) \times m}\end{array}\right]$. Moreover, there exist matrices $\tilde{F} \in \mathbb{R}\left[\mathbf{D}^{\gamma}\right]^{(n-m) \times n}$ and $R \in \mathbb{R}\left[\mathbf{D}^{\gamma}\right]^{m \times n}$ such that System (22) is equivalent to $R x=u, \tilde{F} x=0$.

Theorem 3 If $B$ is hyper-regular, the following statements are equivalent [37]:

(i) System (22) is fractionally 0-flat;

(ii) The system module

$\mathfrak{M}_{\tilde{F}} \triangleq \mathbb{R}\left[\mathbf{D}^{\gamma}\right]^{1 \times n} / \mathbb{R}\left[\mathbf{D}^{\gamma}\right]^{1 \times(n-m)} \tilde{F}$

is free, with $\tilde{F}$ defined in Lemma 1;

(iii) $\tilde{F}$ is hyper-regular over $\mathbb{R}\left[\mathbf{D}^{\gamma}\right]^{(n-m) \times n}$.

\section{Algorithm 2 Fractionally 0-flat output computation}

Input: The matrices $A$ and $B$ of System (22) with $B$ hyper-regular.

Output: Defining matrices $P$ and $Q$ satisfying (25). Procedure:

1. Using row-reduction, check if $B$ is hyper-regular. If not, return "fail".

2. Else, find $M \in G L_{n}\left(\mathbb{R}\left[\mathbf{D}^{\gamma}\right]\right)$ such that $M B=$ $\left[\begin{array}{c}I_{m} \\ 0_{(n-m) \times m}\end{array}\right]$.

3. Obtain $R \in \mathbb{R}\left[\mathbf{D}^{\gamma}\right]^{m \times n}$ and $\tilde{F} \in \mathbb{R}\left[\mathbf{D}^{\gamma}\right]^{(n-m) \times n}$, according to Lemma 1, by: $M A=\left[\begin{array}{c}R \\ \tilde{F}\end{array}\right]$.

4. Find $W \in G L_{n}\left(\mathbb{R}\left[\mathbf{D}^{\gamma}\right]\right)$ according to Proposition 1 (i), such that $\tilde{F} W=\left[I_{n-m}, 0_{(n-m) \times m}\right]$. We therefore get

$Q_{1}=W\left[\begin{array}{c}I_{n-m} \\ 0_{m \times(n-m)}\end{array}\right]$

and

$P_{1}=\left[I_{n-m, 0_{(n-m) \times m}}\right] W^{-1}$.

5. Set $P=\left[P_{1}, 0_{m}\right]$ and $Q=\left[\begin{array}{c}Q_{1} \\ R Q_{1}\end{array}\right]$.

Algorithm 2 gives a fractionally 0-flat output $y=$ $P_{1} x . x=Q_{1} y$ and $u=R Q_{1} y$ identically satisfy (22). 


\subsection{Motion planning}

The nominal transfer function for $\ell=10 \mathrm{~mm}$, as given in Table 1 , is normalized as

$$
\frac{\left(1.1210^{-5} s^{0.73}+2.3510^{-7}\right) e^{-2 s}}{s^{2.19}+0.50 s^{1.46}+2.1610^{-2} s^{0.73}+3.8510^{-5}},
$$

and put under a state-space form:

$\boldsymbol{A}_{\nu} X=B \varphi, \quad T\left(x_{10 m m}, s\right)=C X$,

where

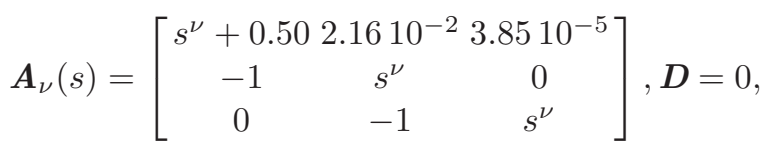

$\boldsymbol{B}=\left[\begin{array}{lll}1 & 0 & 0\end{array}\right]^{T}, \boldsymbol{C}=\left[\begin{array}{llll}0 & 1.1210^{-5} & 2.35 & 10^{-7}\end{array}\right]$.

\section{Flat output computation}

Now apply Algorithm 2. As $\boldsymbol{B}$ is already in its Smith form, introduce $R \in \mathbb{R}\left[s^{\nu}\right]^{1 \times 3}$ and $\tilde{F} \in \mathbb{R}\left[s^{\nu}\right]^{2 \times 3}$ such that : $\boldsymbol{A}_{\nu}=\left[\begin{array}{c}R \\ \tilde{F}\end{array}\right]$ with

$R=\left[s^{\nu}+0.502 .1610^{-2} 3.8510^{-5}\right]$,

$\tilde{F}=\left[\begin{array}{ccc}-1 & s^{\nu} & 0 \\ 0 & -1 & s^{\nu}\end{array}\right]$.

To get the required implicit form, consider the unimodular matrix $M=\left[\begin{array}{l}M_{R} \\ M_{\tilde{F}}\end{array}\right]$ with $M_{R}=\left[\begin{array}{lll}1 & 0 & 0\end{array}\right]$ and $M_{\tilde{F}}=\left[\begin{array}{ll}0_{2 \times 1} & I_{2}\end{array}\right]$ and it can be verified that $M \boldsymbol{A}_{\nu}=$ $\left[\begin{array}{c}R \\ \tilde{F}\end{array}\right], \tilde{F}$ being hyper-regular and thus system (26)-(27) is 0-flat. Let us compute the upper triangular matrix $W \in G L_{3}\left(\mathbb{R}\left[s^{\nu}\right]\right)$ satisfying $\tilde{F} W=\left[I_{3} 0_{2 \times 1}\right]$, with

$W=\left[\begin{array}{ccc}-1 & -s^{\nu} & -s^{2 \nu} \\ 0 & -1 & -s^{\nu} \\ 0 & 0 & -1\end{array}\right]$

and its inverse, also upper-triangular,

$W^{-1}=\left[\begin{array}{ccc}-1 & -s^{\nu} & 0 \\ 0 & -1 & -s^{\nu} \\ 0 & 0 & -1\end{array}\right]$

Therefore,

$Q_{1}=W\left[\begin{array}{l}0 \\ 0 \\ 1\end{array}\right]=\left[\begin{array}{c}-s^{2 \nu} \\ -s^{\nu} \\ -1\end{array}\right]$

$P_{1}=\left[\begin{array}{lll}0 & 0 & 1\end{array}\right] W^{-1}=\left[\begin{array}{lll}0 & 0 & -1\end{array}\right]$,

and one indeed has: $P_{1} Q_{1}=1$.
Finally, the defining matrices $P$ and $Q$ read:

$P=\left[\begin{array}{ll}P_{1} & 0\end{array}\right], \quad Q=\left[\begin{array}{c}Q_{1} \\ R Q_{1}\end{array}\right]$

which proves that system (26)-(27) is fractionally 0-flat and that a flat output $Y$ is given by

$$
\begin{aligned}
Y & =P_{1} X=X_{3} \\
\varphi & =R Q_{1} Y \\
T(\ell=10 m m, s) & =C X=C Q_{1} Y .
\end{aligned}
$$

\section{Trajectory planning}

For a reference temperature trajectory defined for the nominal plant, flatness principles are applied to determine the reference input that generates the reference trajectory. This temperature motion planning is defined with an interpolation polynomial of degree 5: first and second derivatives are nil at the beginning and at the end of the experiment and the temperature should rise from $20^{\circ} \mathrm{C}$ above the ambiant temperature in 1250 s and stabilize for the same duration:

$$
\begin{aligned}
T_{r e f}(t)=T_{i} & +80\left(T_{f}-T_{i}\right) \frac{t^{3}}{t_{f}^{3}} \\
& -240\left(T_{f}-T_{i}\right) \frac{t^{4}}{t_{f}^{4}}+192\left(T_{f}-T_{i}\right) \frac{t^{5}}{t_{f}^{5}},
\end{aligned}
$$

with $T_{i}=0^{\circ} C, T_{f}=20^{\circ} C$, and $t f=2500 \mathrm{~s}$.

The reference output $\tilde{T}_{\text {ref }}(s)$, Laplace transform of $T_{\text {ref }}(t)$, can be expressed from the fractionally flat output $Y$ :

$\tilde{T}_{r e f}=C X$,

with $X=Q_{1} Y$.

$C Q_{1}=1.1210^{-5} s^{\nu}+2.3510^{-7}$ being a polynomial in $s^{\nu}$, its left inverse is not a polynomial :

$\left(C Q_{1}\right)^{-1}=\frac{1}{C Q_{1}}$.

Therefore $Y=\frac{1}{C Q_{1}} T_{\text {ref }}$ and the reference input heat density $\varphi_{\text {ref }}=R Q_{1} Y$.

Open-loop tests on the test bench

Figure 5 presents the results obtained for the nominal model, namely the input reference $\varphi_{\text {ref }}$, the fractionally flat output $Y$, and the effective output compared to the reference trajectory.

As expected, the measured temperature follows the desired temperature and the output error is less than $2 \%$ (less than $0.4^{\circ}$ for a global variation of $20^{\circ}$ ). 

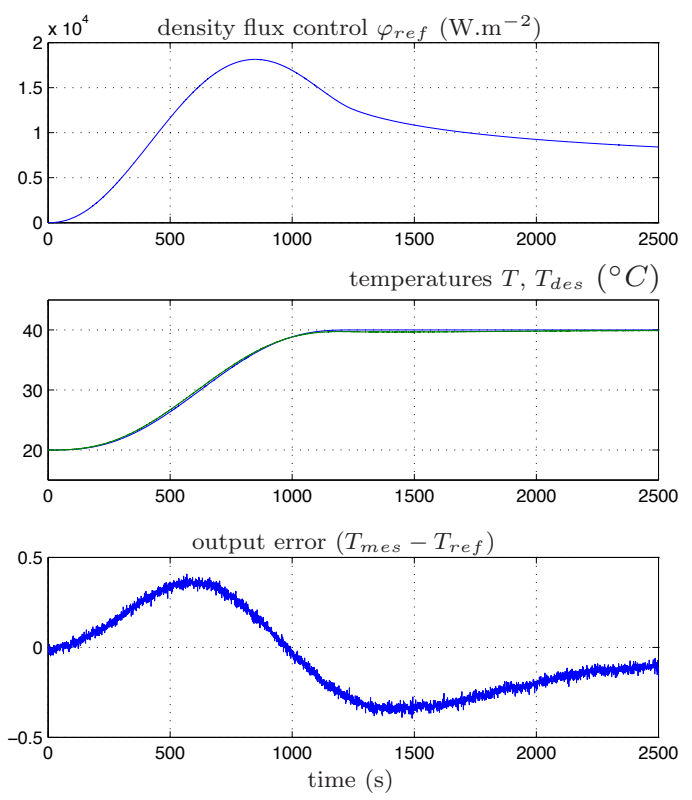

Fig. 5 Open-loop test for the nominal plant : heat flux control $\varphi_{\text {ref }}$, reference $(--)$ and measured (--) outputs, and output error

\subsection{Temperature robust path tracking}

The system considered being fractional and commensurate, a controller adapted to FOS is considered. Openloop tests have been carried out with different sensor positions by applying the reference heat density control. This reference input is only suited for the nominal process (see Figure 6). Also, this open-loop reference control is not adapted for a process other than the nominal one. It is therefore necessary to synthesize a closed-loop control in order to maintain stability degree and the robust path tracking towards parameter uncertainties, modeling errors ans input/output disturbances.

The unit gain frequency $\omega_{u}$ is fixed to $0.12 \mathrm{rad} / \mathrm{s}$ knowing that the sampling frequency is $12.5 \mathrm{rad} / \mathrm{s}$. The controllers should guarantee a phase margin of $50^{\circ}$.

CRONE synthesis The CRONE controller is defined in the frequency band $\left[\omega_{A}, \omega_{B}\right]=[0.01,1] \mathrm{rad} / \mathrm{s}$ around $\omega_{u}$. The third generation CRONE controller should ensure the stability degree robustness towards model parameter variations knowing that at $\omega_{u}$, these variations lead to $[1-9] \mathrm{dB}$ gain variations and to $\left[6.5^{\circ}-90^{\circ}\right]$ phase variations. For security reasons, the density flux control must not exceed $66348 \mathrm{~W} . \mathrm{m}^{-2}$ (10V for the resistor).

The generalized open-loop template is defined as a complex non integer order integrator $n$ whose real part

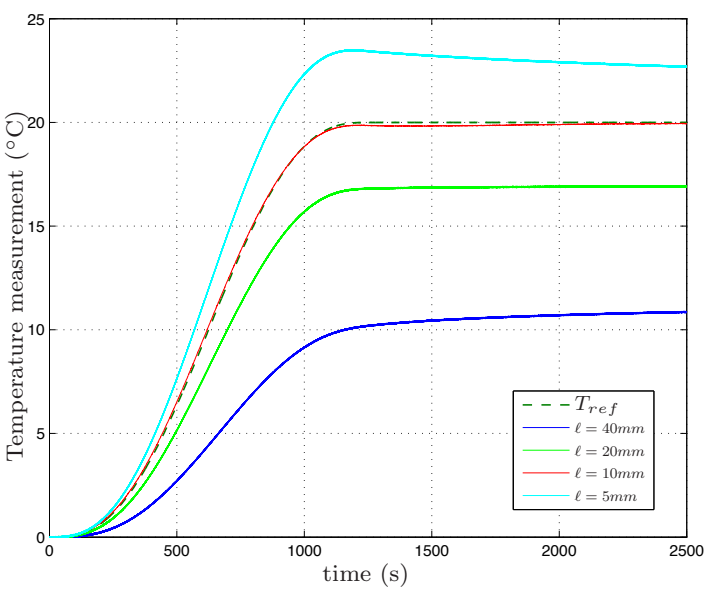

Fig. 6 Time responses of the different models in open-loop

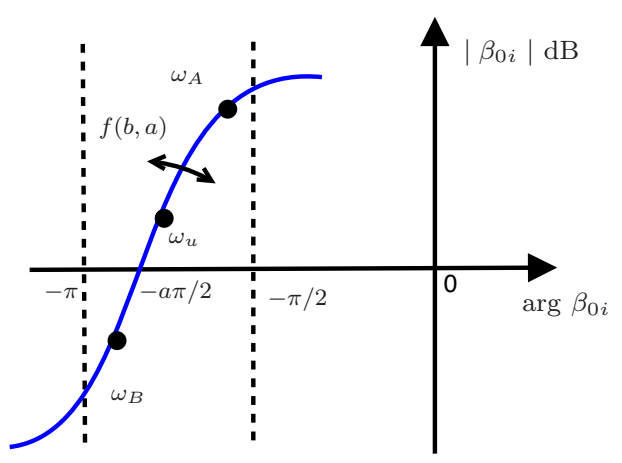

Fig. 7 Generalized template in the Black-Nichols diagram

defines the phase position at $\omega_{u}$, namely $\mathscr{R} e / i(n) \frac{\pi}{2}$, and whose imaginary part defines the angle towards the vertical axis (see Figure 7). The CRONE control methodology is well described in [30].

The open-loop transfer function $\beta_{0}(s)$, including the complex non integer order integration is written as:

$\beta_{0}(s)=C^{\operatorname{sign}(b)}\left(\frac{1+s / \omega_{\mathrm{h}}}{1+s / \omega_{1}}\right)^{a} \mathscr{R} e_{/ \mathrm{i}}\left\{\left(C_{\mathrm{g}} \frac{1+s / \omega_{\mathrm{h}}}{1+s / \omega_{1}}\right)^{\mathrm{i} b}\right\}^{-\operatorname{sign}(b)}$

with $n=a+i b, C_{\mathrm{g}}=\left(\frac{1+\left(\frac{\omega_{\mathrm{u}}}{\omega_{1}}\right)^{2}}{1+\left(\frac{\omega_{\mathrm{u}}}{\omega_{\mathrm{h}}}\right)^{2}}\right)^{1 / 2}$ and $C=$ $\cosh \left[b\left(\arctan \left(\frac{\omega_{\mathrm{u}}}{\omega_{1}}\right)-\arctan \left(\frac{\omega_{\mathrm{u}}}{\omega_{\mathrm{h}}}\right)\right)\right]$. 


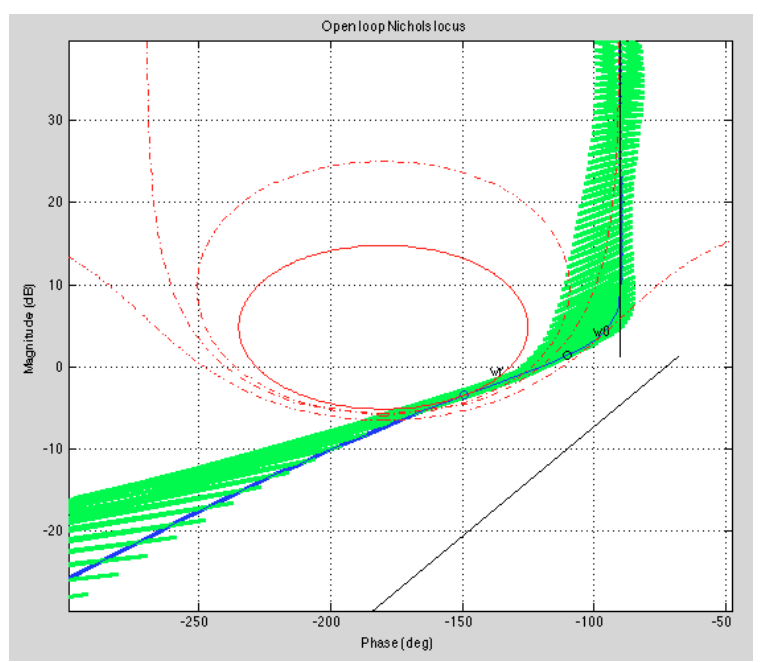

Fig. 8 Nichols chart of the open-loop nominal model (-) and the uncertainties $(\square)$

The nominal model has a delay $\tau$ that must be taken into account in the open-loop transfer function $\beta(s)$ :

$\beta(s)=\beta_{\mathrm{l}}(s) \beta_{0}(s) \beta_{\mathrm{h}}(s) \times e^{-\tau s}$,

with $\tau=2, \nu=0.53, \beta_{1}(s)=C_{1}\left(\frac{\omega_{1}}{s}+1\right)^{n_{1}}$ the order $n_{\mathrm{l}}$ fixing the precision in closed-loop, and $\beta_{\mathrm{h}}(s)=$ $\frac{C_{\mathrm{h}}}{\left(\frac{s}{\omega_{\mathrm{h}}}+1\right)^{n_{\mathrm{h}}}}$ the order $n_{\mathrm{h}}$ enabling the controller to be proper.

The open-loop $\beta(s)$ must tangent an iso-contour in the Nichols chart in order to maintain a low variation of the system stability degree in closed-loop. The optimization of the diagonal open-loop transfer matrix $\beta(s)$ leads to an optimal open-loop in the Nichols chart (see Figure 8) where $\omega_{l}=0.07 \mathrm{rad} / \mathrm{s}, \omega_{h}=1.4 \mathrm{rad} / \mathrm{s}$, $n_{h}=2.46, n_{l}=1, a=1.64$ and $b=-1.27$.

From the nominal open-loop transfer, the fractional controller $C_{F}(s)$ is defined by its frequency response:

$C_{F}(j \omega)=\frac{\beta(j \omega)}{G(j \omega, \ell=10)}$,

where $G(j \omega, \ell=10)$ is the frequency response of the nominal model.

The transfer function of the CRONE third generation controller is then defined by:

$C_{F}(s)=\beta(s) G^{-1}\left(s^{\nu}, \ell=10\right)$.

The synthesis of the rational controller $C_{R}(s)$ is obtained by fitting the frequency response $C_{F}(j \omega)$ with the "Control System Design" module of the CRONE toolbox, whose expression is :

$C_{R}(s)=\frac{6.4310^{9} s^{6}+7.1610^{10} s^{5}+2.0110^{11} s^{4} \ldots}{1.2410^{7} s^{8}+4.1810^{8} s^{7}+4.7210^{8} s^{6} \ldots}$
$\ldots+1.3610^{10} s^{3}+1.4510^{8} s^{2}+1.1110^{5} s+10$
$\ldots+1.8910^{8} s^{5}+3.0310^{7} s^{4}+1.4710^{6} s^{3}+4738 s^{2}+s$

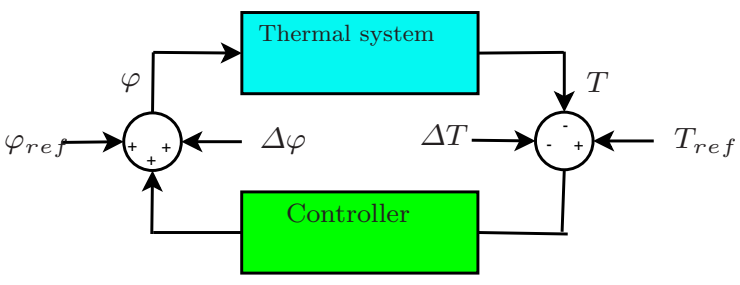

Fig. 9 Closed-loop control scheme

PID controller design The CRONE controller is compared with a PID controller. The specifications of the nominal process $G\left(s^{\nu}, \ell=10 \mathrm{~mm}\right)$ lead to a filtered PID controller synthesized at the same unit gain frequency $\omega_{u}=0.12 \mathrm{rad} / \mathrm{s}$ as:

$C_{P I D}(s)=C_{0} \frac{1+\frac{s}{\omega_{i}}}{\frac{s}{\omega_{i}}} \frac{1+\frac{s}{\omega_{a}}}{1+\frac{s}{\omega_{b}}} \frac{1}{1+\frac{s}{\omega_{f}}}$,

where $C_{0}=22081, \omega_{i}=0.01 \mathrm{rad} / \mathrm{s}, \omega_{a}=0.220 \mathrm{rad} / \mathrm{s}$, $\omega_{b}=0.045 \mathrm{rad} / \mathrm{s}$ and $\omega_{f}=1 \mathrm{rad} / \mathrm{s}$.

\section{Experimental closed-loop tests}

The control scheme is presented in Figure 9: $\varphi_{\text {ref }}$ is the nominal reference control which was obtained through the flatness principles by using the reference temperature trajectory $T_{\text {ref }}$. A control disturbance $\Delta \varphi$ is applied at $625 \mathrm{~s}$ : the cork on the resistor is removed so that a part of the density flux part is dissipated in the air instead of flowing into the rod and a fan is activated to improve the dissipation. Another output disturbance $\Delta T$ is applied at $1875 \mathrm{~s}$ in steady-state: a heating resistor generating $12 \mathrm{~W}$ power (corresponding to variations of $2^{\circ} \mathrm{C}$ ) is glued alongside the rod.

This disturbance scenario is held for different sensor positions and for each PID and CRONE controller: on Figure 10 for $G\left(s^{\nu}, \ell=5 \mathrm{~mm}\right)$, on Figure 11 for $G\left(s^{\nu}, \ell=10 \mathrm{~mm}\right)$, on Figure 12 for $G\left(s^{\nu}, \ell=20 \mathrm{~mm}\right)$ and on Figure 13 for $G\left(s^{\nu}, \ell=40 \mathrm{~mm}\right)$.

The comparison between PID and CRONE controllers on the nominal model show similar responses (see Figure 11). For the other sensor positions, the PID controller is not robust and the path tracking is not efficient. The precision is poorer as the sensor is farther from the heated end. Contrary to a PID controller, a robust path tracking is achieved with a CRONE controller despite the input/output disturbances and the model parametric variations.

\section{Conclusion}

The originality of this paper is in applying system identification, open-loop motion planning and robust con- 

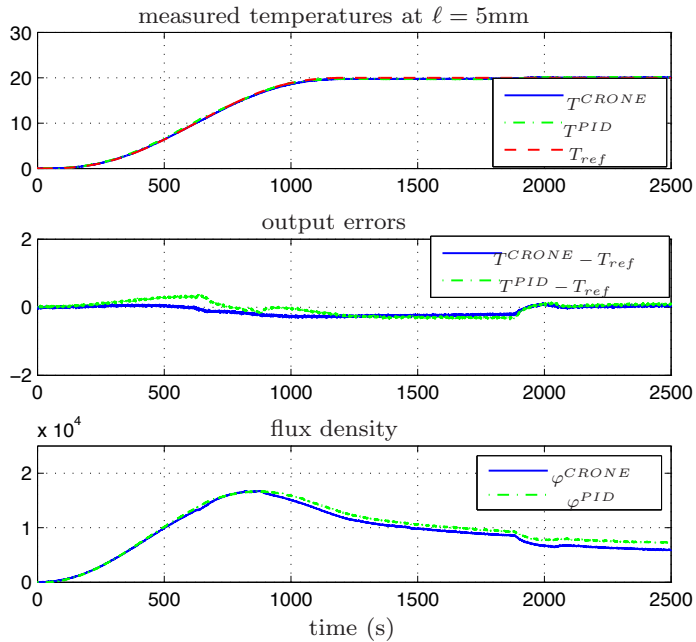

Fig. 10 Measures with disturbances on $G\left(s^{\nu}, \ell=5\right)$ : measured temperatures $T^{C R O N E}(-), T^{P I D}(-.-)$ and the reference $T_{r e f}(--)$, output errors $T^{C R O N E}-T_{r e f}(-)$ and $T^{P I D}-T_{r e f}(-.-)$, and flux density control $\varphi^{C R O N E}(-)$ and $\varphi^{P I D}(-.-)$
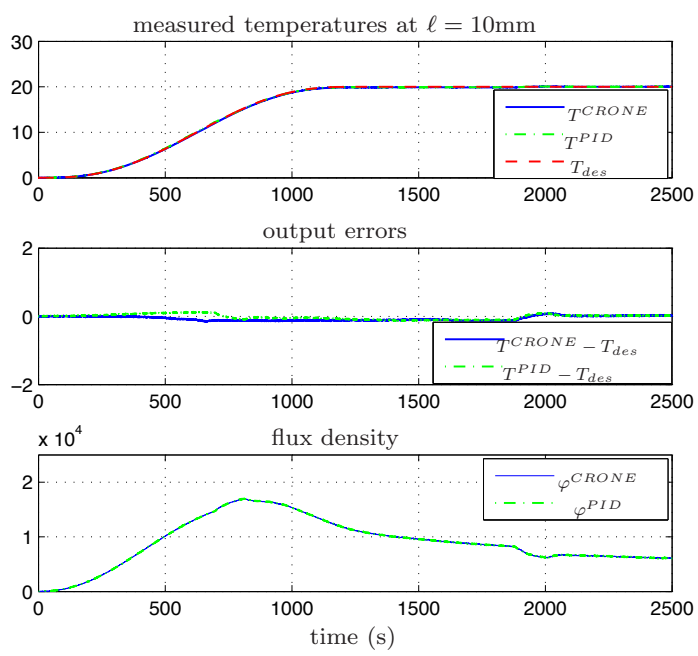

Fig. 11 Measures with disturbances on $G\left(s^{\nu}, \ell=10\right):$ measured temperatures $T^{C R O N E}(-), T^{P I D}(-.-)$ and the reference $T_{\text {ref }}(--)$, output errors $T^{C R O N E}-T_{\text {des }}(-)$ and $T^{P I D}-T_{\text {ref }}(-.-)$, and flux density control $\varphi^{C R O N E}(-)$ and $\varphi^{P I D}(-.-)$

trol to a real heat experiment: the system considered is the model linking the heat transfer throughout an aluminium rod. Temperature sensor positions produce parameter uncertainties. Also, in motion planning, fractionally flat outputs enable to design a reference input in order to follow a desired reference temperature trajectory. Generally, a reference input should be generated for each model. Thanks to a third generation ro-
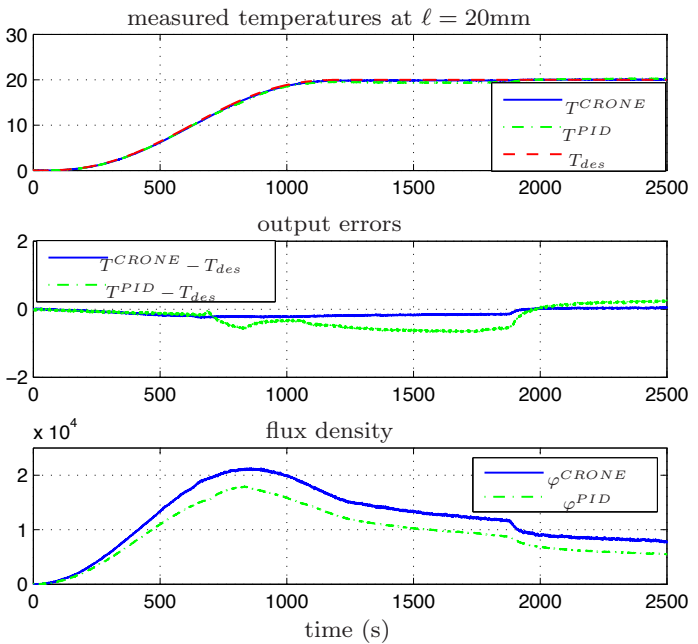

Fig. 12 Measures with disturbances on $G\left(s^{\nu}, \ell=20\right)$ : measured temperatures $T^{C R O N E}(-), T^{P I D}(-.-)$ and the reference $T_{\text {ref }}(--)$, output errors $T^{C R O N E}-T_{\text {ref }}(-)$ et $T^{P I D}-T_{\text {ref }}(-.-)$, and flux density control $\varphi^{C R O N E}(-)$ and $\varphi^{P I D}(-.-)$
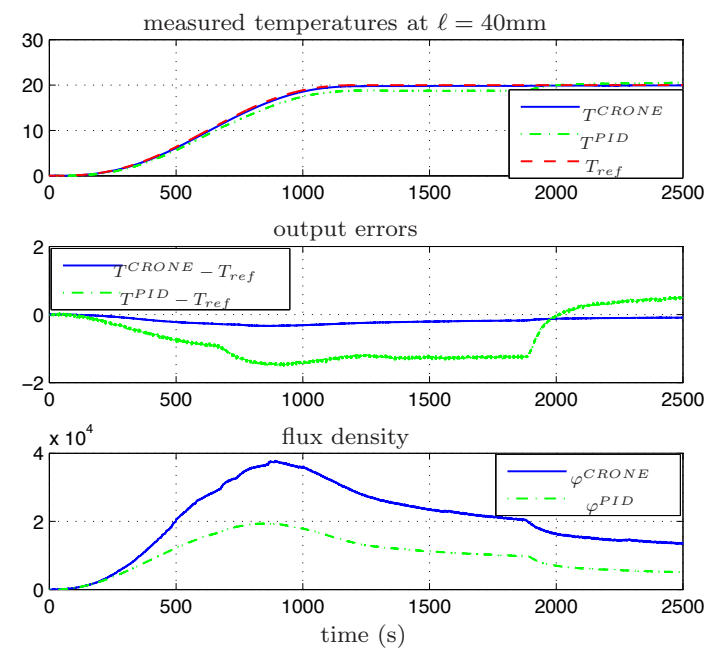

Fig. 13 Measures with disturbances on $G\left(s^{\nu}, \ell=40\right)$ : measured temperatures $T^{C R O N E}(-), T^{P I D}(-.-)$ and the reference $T_{r e f}(--)$, output errors $T^{C R O N E}-T_{r e f}(-)$ et $T^{P I D}-T_{\text {ref }}(-.-)$, and flux density control $\varphi^{C R O N E}(-)$ and $\varphi^{P I D}(-.-)$

bust CRONE controller, it is sufficient to synthesize a unique controller to follow a reference temperature trajectory with the help of the nominal reference control. Moreover, this controller keeps the degree of stability, ensuring robust motion planning by flatness principles and input/output disturbance rejection. A comparison with a PID controller has been carried out and results have been really convincing. 


\section{References}

1. Amini, A., Azarbahram, A., Sojoodi, M.: $H_{\infty}$ consensus of nonlinear multi-agent systems using dynamic output feedback controller: an LMI approach. Nonlinear Dynamics pp. 1-22 (2016). DOI 10.1007/s11071-016-2801-6

2. Antritter, F., Cazaurang, F., Lévine, J., Middeke, J.: On the computation of $\pi$-flat outputs for differential-delay linear systems. Systems \& Control Letters 71, 14-22 (2014). DOI 10.1016/j.sysconle.2014.07.002

3. Antritter, F., Middeke, J.: A toolbox for the analysis of linear systems with delays. In: CDC/ECC 2011, pp. 19501955. Orlando, USA (2011)

4. Aoun, M., Malti, R., Levron, F., Oustaloup, A.: Synthesis of fractional Laguerre basis for system approximation. Automatica 43, 1640-1648 (2007). DOI 10.1016/j.automatica.2007.02.013

5. Battaglia, J.L., Le Lay, L., Batsale, J.C., Oustaloup, A., Cois, O.: Heat flux estimation through inverted non integer identification models. International Journal of Thermal Science 39(3), 374-389 (2000). DOI 10.1016/S12900729(00)00220-9

6. Battig, A., Kalla, S.: On Mikusinski's operators of fractional integration. Revista Colombiana de Matemáticas IX, 155-160 (1975)

7. Benchellal, A., Bachir, S., Poinot, T., Trigeassou, J.C.: Identification of a non-integer model of induction machines. In: 1st IFAC Workshop on Fractional Differentiation and its Applications (FDA), pp. 400-407. Bordeaux, France (2004)

8. Brunovský, P.: A classification of linear controllable systems. Kybernetika 6, 176-178 (1970)

9. Cohn, P.M.: Free rings and Their relations. London, Academic Press (1985)

10. Cois, O., Oustaloup, A., Poinot, T., Battaglia, J.L.: Fractional state variable filter for system identification by fractional model. In: 6th European Control Conference ECC'01. Porto, Portugal (2001)

11. Farges, C., Fadiga, L., Sabatier, J.: $H_{\infty}$ analysis and control of commensurate fractional order systems. Mechatronics 23(7), 772-780 (2013). DOI 10.1016/j.mechatronics.2013.06.005

12. Fliess, M.: Some basic structural properties of generalized linear systems. Systems Control Letters 15, 391-396 (1990). DOI 10.1016/0167-6911(90)90062-Y

13. Fliess, M., Hotzel, R.: Sur les systèmes linéaires à dérivation non entière. Comptes Rendus de l'Académie des Sciences - Series IIB - Mechanics-Physics-ChemistryAstronomy 324(2), 99 - 105 (1997). DOI 10.1016/S12518069(99)80013-X

14. Fliess, M., Lévine, J., Martin, P., Rouchon, P.: Sur les systèmes non linéaires différentiellement plats. Comptes rendus de l'Académie des sciences I-315, 619-624 (1992)

15. Gabano, J.D., Poinot, T., Kanoun, H.: Identification of a thermal system using continuous linear parameter-varying fractional modelling. IET Control Theory \& Applications 5(7), 889-899 (2011). DOI 10.1049/iet-cta.2010.0222

16. Garnier, H., Wang, L.: Identification of continuous-time models from sampled data. Springer-Verlag (2008)

17. Heymans, N., Bauwens, J.: Fractal rheological models and fractional differential equations for viscoelastic behavior. Rheologica Acta 33, 219 (1994). DOI 10.1007/BF00437306

18. Kailath, T.: Linear Systems. Prentice-Hall, Englewood Cliffs, NJ (1980)

19. Lamara, A., Colin, G., Lanusse, P., Charlet, A., NelsonGruel, D., Chamaillard, Y.: Pollutant reduction of a turbocharged diesel engine using a decentralized MIMO CRONE controller. Fractional Calculus and Applied Analysis 18(2), 307-332 (2015). DOI 10.1515/fca-2015-0021

20. Lévine, J.: Analysis and Control of Nonlinear Systems A Flatness-based Approach. Springer Berlin Heidelberg (2009)

21. Lévine, J., Nguyen, D.V.: Flat output characterization for linear systems using polynomial matrices. System \& Control Letters 48, 69-75 (2003). DOI 10.1016/S01676911(02)00257-8

22. Ljung, L.: System identification - Theory for the user, 2 edn. Prentice-Hall, Upper Saddle River, N.J., USA (1999)

23. Maachou, A., Malti, R., Melchior, P., Battaglia, J., Oustaloup, A., Hay, B.: Nonlinear thermal system identification using fractional Volterra series. Control Engineering Practice 29, 50-60 (2014). DOI 10.1016/j.conengprac.2014.02.023

24. Malti, R., Sabatier, J., Akçay, H.: Thermal modeling and identification of an aluminium rod using fractional calculus. In: 15th IFAC Symposium on System Identification (SYSID'2009), pp. 958-963. St Malo, France (2009). DOI 10.3182/20090706-3-FR-2004.00159

25. Malti, R., Victor, S., Oustaloup, A.: Advances in system identification using fractional models. Journal of Computational and Nonlinear Dynamics 3, 021,401.1-021,401.7 (2008). DOI 10.1115/1.2833910

26. Matignon, D., d'Andréa-Novel, B.: Some results on controllability and observability of finite-dimensional fractional differential systems. In: IMACS, vol. 2, pp. 952-956. IEEESMC, Lille, France (1996)

27. Mikusiński, J.: Operational Calculus. Pergamon, Oxford \& PWN, Varsovie (1983)

28. Miller, K., Ross, B.: An introduction to the fractional calculus and fractional differential equations. A WileyInterscience Publication (1993)

29. Oldham, K., Spanier, J.: The remplacement of Fick's laws by a formulation involving semi-differentiation. Journal of Electroanalytical Chemistry and Interfacial Electrochemistry 26(2-3), 331-341 (1970). DOI 10.1016/S00220728(70)80316-3

30. Oustaloup, A.: La commande CRONE. Hermès - Paris (1991)

31. Podlubny, I.: Fractional Differential Equations. Academic Press, San Diego (1999)

32. Polderman, J., Willems, J.: Introduction to Mathematical System Theory: a behavioral approach. Springer-Verlag, Berlin (1998)

33. Qian, S., Zi, B., Ding, H.: Dynamics and trajectory tracking control of cooperative multiple mobile cranes. Nonlinear Dynamics 83(1), 89-108 (2016). DOI 10.1007/s11071-0152313-9

34. Rodrigues, S., Munichandraiah, N., Shukla, A.K.: A review of state of charge indication of batteries by means of A.C. impedance measurements. Journal of Power Sources 87(1-2), 12-20 (2000). DOI 10.1016/S0378-7753(99)003511

35. Sabatier, J., Merveillaut, M., Francisco, J., Guillemard, F., Porcelatto, D.: Lithium-ion batteries modeling involving fractional differentiation. Journal of power sources 262, 3643 (2014). DOI 10.1016/j.jpowsour.2014.02.071

36. Victor, S., Malti, R., Garnier, H., Oustaloup, A.: Parameter and differentiation order estimation in fractional models. Automatica 49(4), 926-935 (2013). DOI 10.1016/j.automatica.2013.01.026

37. Victor, S., Melchior, P., Lévine, J., Oustaloup, A.: Flatness for linear fractional systems with application to a thermal system. Automatica 57, 213-221 (2015). DOI 10.1016/j.automatica.2015.04.021 
38. Young, P., Jakeman, A.: Refined instrumental variable methods of time-series analysis: Part III, extensions. International Journal of Control 31, 741-764 (1980). DOI $10.1080 / 00207178008961080$ 